\title{
Failure Analyses of Three 6061-T6 Aluminum alloy Turbo-Expander Wheels Exposed to Natural Gas Environments,
}

\author{
Behzad Bavarian, PhD \\ Dept. of Manufacturing System Engineering and Management \\ California State University, Northridge 91330-8332
}

Failure analyses were performed on three fractured 6061-T6 Aluminum alloy turbo-expander wheels (\#A, \#B, \& \#C) that were exposed to mercury-bearing natural gas environment, using Scanning Electron Microscopy (SEM), Energy Dispersive Analysis(EDAX), optical microscopy, and mechanical tests to determine the cause of their premature failures.

This investigation showed that causes of these wheel failures were corrosion attacks byliquid metal embrittlement (LME) of the 6061 Al-alloy by mercury. Mercury corroded and embrittled the 6061T6 Al-alloy resulting in several crack initiations at the edges of several blades, and inside expander wheel holes. LME also caused a loss of wheel toughness, which led to corrosion fatigue and stress corrosion cracking led to their premature failure of these wheels.

SEM fractographs of these wheels showed several fatigue crack initiation sites located at severely corroded regions on the edges of their blades, and EDAX analyses showed a presence of large amount of mercury, chloride and sulfide at these locations. The fracture surfaces were relatively flat, indicating the absence of an appreciable amount of gross plastic deformation, unusual for the ductile 6061-T6 Al-alloy. Clearly lack of toughness was observed for both wheels, brittle cleavage cracking modes, and the presence of a few droplets of mercury on the fracture surfaces strongly suggested that liquid mercury attacked the aluminum and initiated fatigue cracks; LME caused severe toughness loss of the alloy. Presence of some corrosion products and mercury droplets covering the wheel fracture surfaces were observed. These observations indicate that a few cracks were initiated by liquid metal attack, and then propagated until the final stage of fracture. The cleavage like faceted morphology of fatigue striations during the fatigue propagation stages indicated a low stress intensity level during these stages. These observations indicate that the wheel was designed properly for this application; however, severe mercury attacks shortened their lives. The last stage of failure for both wheels showed overloaded failure modes, demonstrating that these wheels possess proper toughness, but mercury embrittlement resulted in their toughness loss.

SEM/EDAX results showed some severe corrosion attacks on both wheels by mercury embrittlement. In addition, large amounts of sulfur and chloride were also detected in the corrosion products present on the fracture surfaces, which could also cause serious corrosion problems for aluminum alloys.

In conclusion, causes of these wheels' failure were corrosion attacks due to liquid mercury embrittlement of 6061 Al-alloy, which resulted in delayed corrosion assisted failures of these wheels. The source of mercury was possibly the flowing gas. The stress level was relatively low which indicates the design of the wheel was appropriate. The presence of some fatigue crack propagation prior to the final fracture of both wheels verified the sound design of these wheels. 


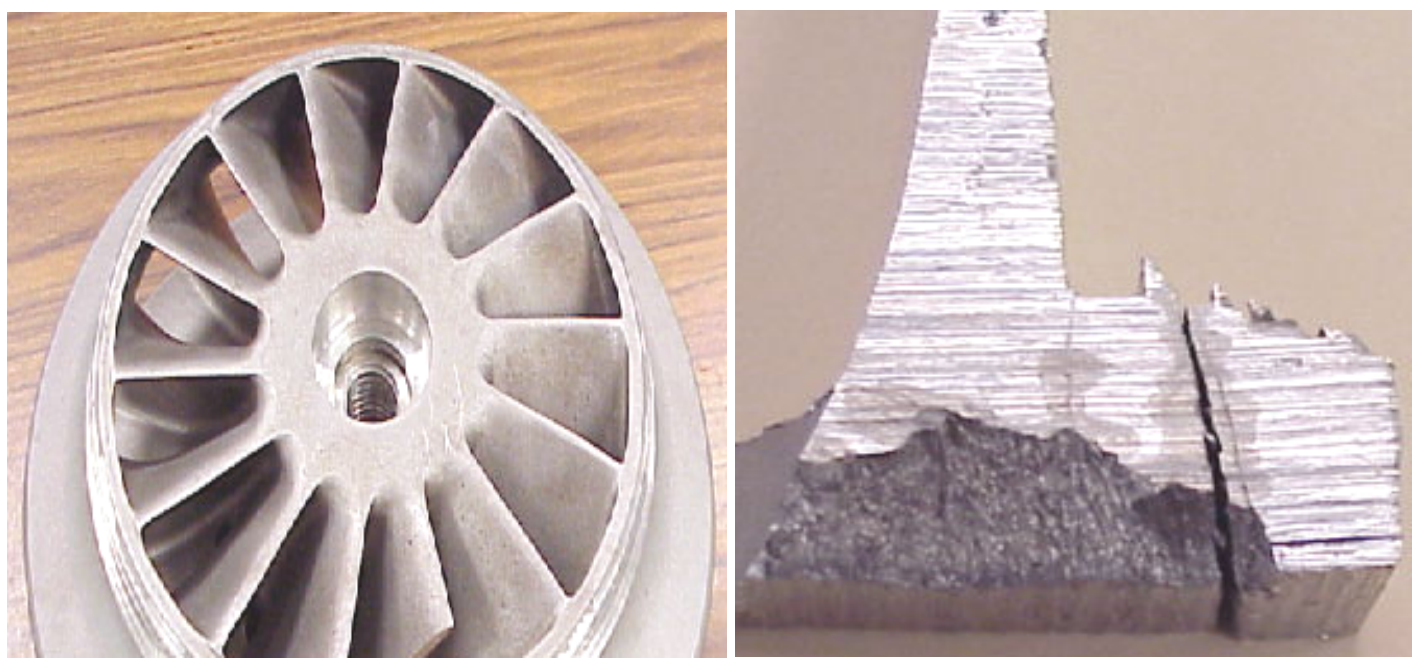

Figure 1: Optical images of the fractured wheel showing a few of secondary cracks that were fractured to analyze their failure mode, Fracture surfaces were covered with $\mathrm{Hg}$ and corrosion attacks.

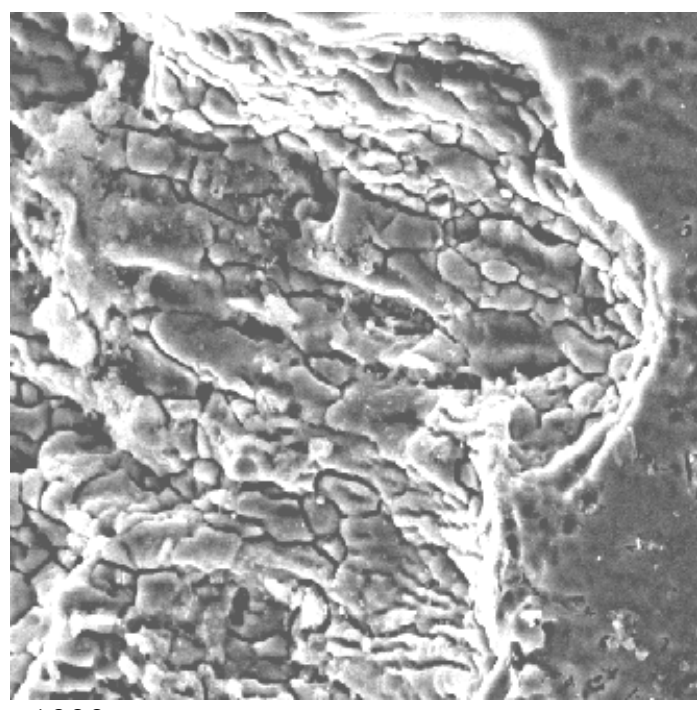

$\mathrm{x} 1000$

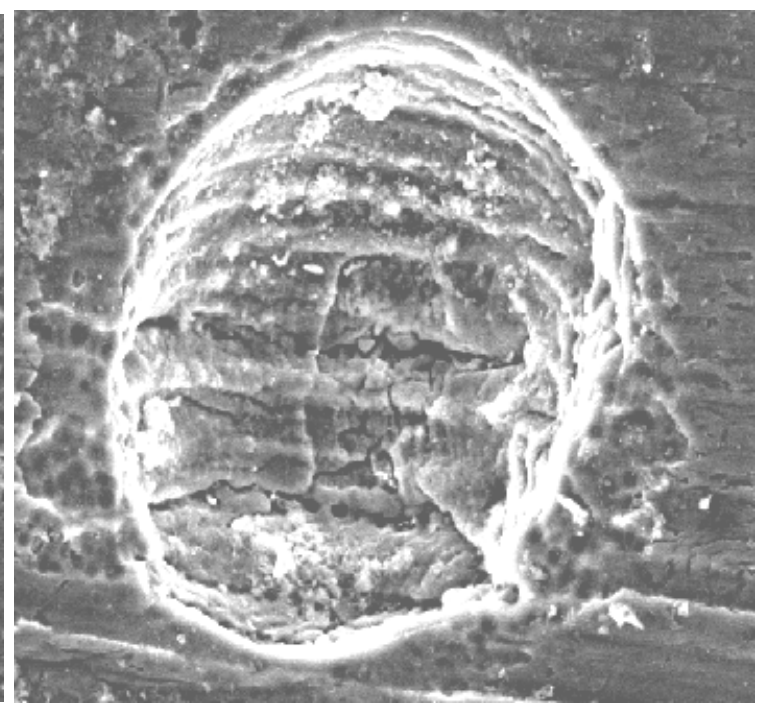

x600

Figure 2: SEM images of the fractured wheel, showing corrosion attacks inside a pit caused by Hg-attack. 